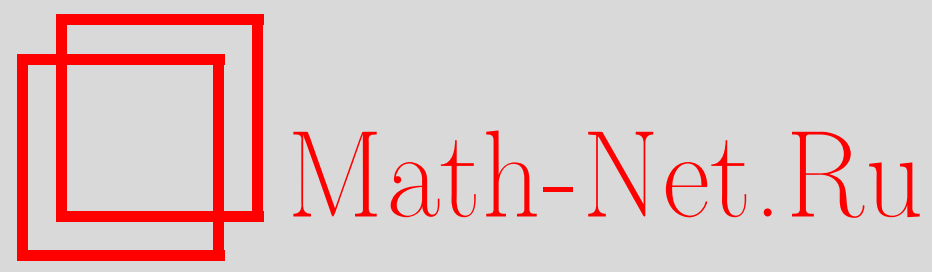

М. Ю. Неклюдов, Производная меры Винера на траекториях в компактной группе Ли, Матем. заметки, 2004, том 75, выпуск 5, 789-792

DOI: https://doi.org/10.4213/mzm558

Использование Общероссийского математического портала Math-Net.Ru подразумевает, что вы прочитали и согласны с пользовательским соглашением http://www . mathnet.ru/rus/agreement

Параметры загрузки:

IP : 3.85 .7 .115

26 апреля 2023 г., $13: 27: 29$

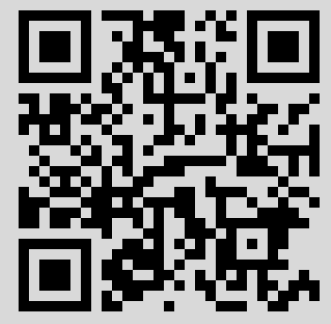




\section{ПРОИЗВОДНАЯ МЕРЫ ВИНЕРА \\ НА ТРАЕКТОРИЯХ В КОМПАКТНОЙ ГРУППЕ ЛИ}

\section{М.Ю. Неклюдов}

Пространством Камерона-Мартина $H$ меры Радона $\mu$, определенной на локально выпуклом пространстве $X$, называется множество направлений $h \in X$, вдоль которых эта мера дифоференцируема. Мера $\mu$ называется дифференцируемой вдоль направления $h$, если сушествует такая функция $\beta_{h}^{\mu} \in L^{1}(\mu)$, что справедлива формула интегрирования по частям:

$$
\int_{X} \partial_{h} f(x) d \mu=-\int_{X} f(x) \beta_{h}^{\mu}(x) d \mu
$$

для любой гладкой цилиндрической функции $f$. При этом функция $\beta_{h}^{\mu}$ называется логарифмической производной меры $\mu$ вдоль $h$. Аналогично определяется дифференцируемость меры вдоль векторного поля $v: X \rightarrow X$ (точные определения приводятся ниже). В статье [1] была доказана дифференцируемость мер вдоль некоторого класса векторных полей, принимающих значение в пространстве Камерона-Мартина. В настоящей работе дано описание класса векторных полей, не принимающих значения в пространстве Камерона-Мартина меры Винера на $C\left([0,1], \mathbb{R}^{n}\right)$ ни в одной точке, вдоль которых эта мера дифференцируема. Поля, значения которых не принадлежат пространству Камерона-Мартина, естественно возникают при рассмотрении мер на пространствах функций со значениями в группах Ли [2]. С помощью доказанного результата описан один класс векторных полей на траекториях в компактной связной группе $G$, вдоль которьх дифференцируема мера Винера $W_{G}^{z}$ на $C([0,1], G)$, и найдена логарифмическая производная вдоль этих полей.

1. Обозначения и терминология. Если $X$ - топологическое пространство, то В $(X)$ - это $\sigma$-алгебра его борелевских подмножеств, $M(X)$ - векторное пространство всех (конечных) счетно-аддитивных мер на $\mathrm{B}(X), C_{b}(X)$ - пространство всех ограниченных непрерьвных вещественных функций на $X$; всякое множество $A \subset X$ считается наделенным топологией, индуцированной топологией пространства $X$. Если $X_{1}$ и $X_{2}$ - топологические пространства, то $C\left(X_{1}, X_{2}\right)$ - это пространство всех непрерывных отображений $X_{1}$ в $X_{2}$, наделенное компактно-открытой топологией. Если $\nu \in M(X)$ и $A \in \mathrm{B}(X)$, то $\nu_{A}$ - это сужение $\nu$ на $\mathrm{B}(A)$, так что $\nu_{A} \in M(A)$.

Пусть $G$ - компактная связная группа Ли, реализованная как подгруппа $O\left(\mathbb{R}^{k}\right) \cong \mathbb{R}^{k^{2}}, g$-ее алгебра Ли. В $\mathbb{R}^{k^{2}}$ введено скалярное произведение $(A, B)=\operatorname{tr} A B^{*}$, инвариантное относительно действия группы $O\left(\mathbb{R}^{k}\right) ; G_{\varepsilon}-\varepsilon$-окрестность $G$ в $\mathbb{R}^{k^{2}}$. Пусть также $\exp (): g \rightarrow G$ - экспоненциальне отображение $g$ в $G$.

Пусть $C_{b}^{\text {cyl }}\left([0,1], \mathbb{R}^{n}\right)$ - пространство всех ограниченных вещественных цилиндрических функций на $C\left([0,1], \mathbb{R}^{n}\right)$ и $\sigma=\sigma\left(C\left([0,1], \mathbb{R}^{n}\right), C_{b}^{\text {cyl }}\left([0,1], \mathbb{R}^{n}\right)\right) ;$ функция $F: C\left([0,1], \mathbb{R}^{n}\right) \in \mathbb{R}^{1}$ называется цилиндрической, если существует $k \in \mathbb{N}, t_{1}, t_{2}, \ldots, t_{k} \in(0,1)$ и $\psi: \mathbb{R}^{k} \rightarrow \mathbb{R}^{1}$ такие, что для всех $g \in C\left([0,1], \mathbb{R}^{n}\right) F(g)=\psi\left(g\left(t_{1}\right), g\left(t_{2}\right), \ldots, g\left(t_{k}\right)\right)$. Аналогично определяется $C_{b}^{\text {cyl }}([0,1], G)-$ пространство всех ограниченных цилиндрических функций на $C([0,1], G)$.

Для всякого $z \in G$ символ $W_{G}^{z}$ обозначает меру Винера на $C([0,1], G)$, сосредоточенную на функциях, принимающих значение $z$ при $t=0$ (определение меры Винера на траекториях в многообразии приведено в [3]); $W_{\mathbb{R}^{n}}^{z}$ - мера Винера на $C\left([0,1], \mathbb{R}^{n}\right)$, сосредоточенная на функциях, принимающих значение $z$ при $t=0 ; C_{z}([0,1], G)=\{x \in C([0,1], G) \mid x(0)=z\}$.

Касательным пространством к $C_{z}([0,1], G)$ в точке $\omega \in C_{z}([0,1], G)$ будем называть множество $T_{\sigma} C_{z}([0,1], G)$ непрерывных векторных полей $X(s)$ вдоль $\omega$ таких, что $X(0)=0 ; \quad T_{\omega} C_{z}([0,1], G)=\left\{X \in C_{z}([0,1], T G) \mid X(s) \in T_{\omega(s)} G \forall s \in[0,1], X(0)=0\right\}$. Объединение касательных пространств во всех точках $\omega \in C_{z}([0,1], G)$ будем обозначать

Работа выполнена при поддержке Российского фонда фундаментальных исследований, грант № 02-01-01074. 
$T\left(C_{z}([0,1], G)\right)=\bigcup_{\omega \in C_{z}([0,1], G)} T_{\omega} C_{z}([0,1], G)$. Векторным полем $v$ на $C_{z}([0,1], G)$ называется непрерывное отображение из топологического пространства $C_{z}([0,1], G)$ в пространство $T\left(C_{z}([0,1], G)\right)$ такое, что $\forall \omega \in C_{z}([0,1], G) \quad v(\omega) \in T_{\omega} C_{z}([0,1], G)$. Векторное поле на $C_{z}\left([0,1], \mathbb{R}^{n}\right)$ определяется аналогично.

2. Основной результат и доказательство. Пусть $u_{\tau}: C_{z}\left([0,1], \mathbb{R}^{n}\right) \rightarrow C_{z}\left([0,1], \mathbb{R}^{n}\right)$, $u_{\tau}(x)(t)=e^{\tau \alpha(t)} x(t), \alpha \in C^{1}([0,1], g), \alpha(0)=0,-$ поток отображений $C_{z}\left([0,1], \mathbb{R}^{n}\right)$. Тогда $v(x)=d u_{\tau} /\left.d \tau\right|_{\tau=0}(x)$ - векторное поле на $C_{z}\left([0,1], \mathbb{R}^{n}\right)$, причем сужение этого поля на $C_{z}([0,1]$, $G)$ тоже является векторным полем.

ОПРЕДЕЛЕНИЕ 1. Будем называть меру $\mu \in M\left(C_{z}([0,1], G)\right)$ дифферениируемой вдоль векторного поля $v: C_{z}([0,1], G) \rightarrow T\left(C_{z}([0,1], G)\right)$, если выполнено равенство

$$
\int_{C_{z}([0,1], G)} \partial_{v} f(x) d \mu=-\int_{C_{z}([0,1], G)} f(x) \beta_{v}^{\mu}(x) d \mu \quad \forall f \in C_{b}^{\mathrm{cyl}}([0,1], G),
$$

при этом функцию $\beta_{v}^{\mu}$ будем называть логарифмической производной меры $\mu$ вдоль векторно2o nоля $v$. Другие определения логарифмической производной, связь между ними, а также использование логарифмических производных для квантования бесконечномерных гамильтоновых систем есть в статьях [1], [4]-[6].

Заметим, что мы рассматриваем класс полей со значениями, не принадлежащими пространству Камерона-Мартина, так как траектории винеровского процесса не дифференцируемы с вероятностью 1 и, следовательно, общие теоремы из статьи [1] в нашем случае не применимы.

TEOPEMa 1. Ec $u \quad f \in C_{b}^{\mathrm{cyl}}\left([0,1], \mathbb{R}^{n}\right) u$

$$
\int_{C\left([0,1], \mathbb{R}^{n}\right)} e^{\tau \int_{0}^{1}(\hat{\alpha}(\tau, s) x(s), d x(s))} W_{\mathbb{R}^{n}}^{z}(d x)<\infty, \quad \text { ¿de } \quad \hat{\alpha}(\tau, s)=e^{\tau \alpha(s)} \dot{\alpha}(s) e^{-\tau \alpha(s)}
$$

для некоторого $\tau>0$, то верно следующее равенство:

$$
\begin{gathered}
\int_{C\left([0,1], \mathbb{R}^{n}\right)} f\left(u_{\tau}(x)\right) W_{\mathbb{R}^{n}}^{z}(d x)=\int f(x) \exp \left(\tau \int_{0}^{1}(\hat{\alpha}(\tau, s) x(s), d x(s))\right. \\
\left.-\frac{1}{2} \tau^{2} \int_{0}^{1}(\hat{\alpha}(\tau, s) x(s), \hat{\alpha}(\tau, s) x(s)) d s\right) W_{\mathbb{R}^{n}}^{z}(d x) \quad \forall f \in C_{b}^{\mathrm{cyl}}\left([0,1], \mathbb{R}^{n}\right)
\end{gathered}
$$

әде $x_{s}$ - винеровский прочесс на $C\left([0,1], \mathbb{R}^{n}\right)$ (относительно меры $\left.W_{\mathbb{R}^{n}}^{z}\right)$.

ДокАЗАТЕЛЬСтво. Заметим, что мера

$$
\widetilde{P}=\exp \left(\tau \int_{0}^{1}(\hat{\alpha}(\tau, s) x(s), d x(s))-\frac{1}{2} \tau^{2} \int_{0}^{1}(\hat{\alpha}(\tau, s) x(s), \hat{\alpha}(\tau, s) x(s)) d s\right) W_{\mathbb{R}^{n}}^{z}
$$

является вероятностной мерой, так как если обозначить $Y_{t}=\tau \int_{0}^{t}(\hat{\alpha}(\tau, s) x(s), d x(s)), M_{t}=$ $e^{Y_{t}-\langle Y\rangle_{t} / 2}$ (здесь $\langle\cdot\rangle$ - квадратичная вариация), то $M_{t}$ является мартингалом по теореме Казамаки $[3$, гл. $3, \S 5$, замечание 5.1$]$ и (так как $\left.\widetilde{P}=M_{1} W_{\mathbb{R}^{n}}^{z}\right)$

$$
\widetilde{P}\left(C\left([0,1], \mathbb{R}^{n}\right)\right)=\int_{C\left([0,1], \mathbb{R}^{n}\right)} M_{1}(x) W_{\mathbb{R}^{n}}^{z}(d x)=\int_{C\left([0,1], \mathbb{R}^{n}\right)} M_{0}(x) W_{\mathbb{R}^{n}}^{z}(d x)=1 .
$$

Будем обозначать интеграл от функции $f$ по этой мере $\widetilde{E} f$. По теореме Гирсанова мера $\widetilde{P}$ получается из меры Винера путем параллельного переноса вдоль поля $\tau \int_{0}^{t} \hat{\alpha}(\tau, s) x(s) d s$. Таким образом 
для доказательства леммы нам достаточно доказать, что $e^{-\tau \alpha(t)} x(t)$ - винеровский процесс относительно меры $\widetilde{P}$. Из теоремы Гирсанова следует, что $x(t)-\tau \int_{0}^{t} \hat{\alpha}(\tau, s) x(s) d s$ является винеровским процессом относительно $\widetilde{P}$, т.е. вьполнено следующее равенство:

$$
\widetilde{E}\left[e^{i\left\langle\zeta, x(t)-x(s)-\tau \int_{s}^{t} \hat{\alpha}(\tau, s) x(s) d s\right\rangle} \mid \mathscr{F}_{s}\right]=e^{-\frac{(t-s)|\zeta|^{2}}{2}} .
$$

Обозначим $y(t)=e^{-\tau \alpha(t)} x(t)$ и перепишем равенство в виде

$$
\widetilde{E}\left[e^{i\left\langle\zeta, e^{\tau \alpha(t)} y(t)-e^{\tau \alpha(s)} y(s)-\tau \int_{s}^{t} e^{\tau \alpha(q)} \dot{\alpha}(q) y(q) d q\right\rangle} \mid \mathscr{F}_{s}\right]=e^{-\frac{(t-s)|\zeta|^{2}}{2}} .
$$

Из формулы Ито следует, что

$$
e^{\tau \alpha(t)} y(t)-e^{\tau \alpha(s)} y(s)-\tau \int_{s}^{t} e^{\tau \alpha(q)} \dot{\alpha}(q) y(q) d q=\int_{s}^{t} e^{\tau \alpha(q)} d y(q)
$$

Таким образом $z(t)=\int_{0}^{t} e^{\tau \alpha(q)} d y(q)-$ броуновское движение относительно $\widetilde{P}$, но так как $e^{\tau \alpha(q)}$ семейство ортогональных матриц, то и $y(t)$ тоже броуновское движение (так как $y(t)$ и $z(t)$ имеют одинаковые квадратичные ковариации). Доказательство закончено.

СледСтвиЕ 1. Если выполнено условие

$$
\int_{C\left([0,1], \mathbb{R}^{n}\right)} e^{\tau \int_{0}^{1}(\hat{\alpha}(\tau, s) x(s), d x(s))} W_{\mathbb{R}^{n}}^{z}(d x)<\infty
$$

для некоторого $\tau>0$, то мера Винера $W_{\mathbb{R}^{n}}^{z}$ дифференцируема вдоль поля $v(x)$ и ее логарифмическая производная равна $\int_{0}^{1}(\hat{\alpha}(\tau, s) x(s), d x(s))_{\mathbb{R}^{n}}$.

ДокАЗАТЕльство. Из теоремы следует, что мера Винера $W_{\mathbb{R}^{n}}^{z}$ дифференцируема вдоль поля $v$ и ее логариффическая производная равна $\int_{0}^{1}(\hat{\alpha}(\tau, s) x(s), d x(s))_{\mathbb{R}^{n}}$, так как

$$
\begin{gathered}
\int\left\langle f^{\prime}(x), v(x)\right\rangle W_{\mathbb{R}^{n}}^{z}(d x)=\lim _{\tau \rightarrow 0} \int \frac{f\left(u_{\tau}(x)\right)-f(x)}{\tau} W_{\mathbb{R}^{n}}^{z}(d x) \\
=\lim _{\tau \rightarrow 0} \int f(x) \frac{e^{\tau \int_{0}^{1}(\hat{\alpha}(\tau, s) x(s), d x(s))-\frac{1}{2} \tau^{2} \int_{0}^{1}(\hat{\alpha}(\tau, s) x(s), \hat{\alpha}(\tau, s) x(s)) d s}-1}{\tau} \\
W_{\mathbb{R}^{n}}^{z}(d x)=\int f(x) \int_{0}^{1}(\hat{\alpha}(\tau, s) x(s), d x(s))_{\mathbb{R}^{n}} W_{\mathbb{R}^{n}}^{z}(d x) .
\end{gathered}
$$

Доказательство закончено.

3. Дифференцируемость меры Винера на функциях со значениями в компактной группе Ли.

TeOpema 2. Ec^u $f \in C_{b}^{\mathrm{cyl}}([0,1], G) u$

$$
\int_{C([0,1], G)} e^{\tau \int_{0}^{1}(\hat{\alpha}(\tau, s) x(s), d x(s))} W_{G}^{z}(d x)<\infty
$$

для некоторого $\tau>0$, то верно следующее равенство:

$$
\begin{aligned}
& \int_{C([0,1], G)} f\left(u_{\tau}(x)\right) W_{G}^{z}(d x)=\int f(x) \exp \left(\tau \int_{0}^{1}(\hat{\alpha}(\tau, s) x(s), d x(s))\right. \\
& \left.-\frac{1}{2} \tau^{2} \int_{0}^{1}(\hat{\alpha}(\tau, s) x(s), \hat{\alpha}(\tau, s) x(s)) d s\right) W_{G}^{z}(d x) \quad \forall f \in C_{b}^{\mathrm{cyl}}([0,1], G),
\end{aligned}
$$

әде $x_{s}$ - винеровский прочесс на $C([0,1], G)$ (относительно меры $\left.W_{G}^{z}\right)$. 
ДокАЗАТЕльство. Ниже используется следующая теорема, сформулированная в статье [2] (доказательство приведено в [7]).

ТЕОРема. Пусть $G$ - компактная группа Ли, вложенная в $O\left(\mathbb{R}^{k}\right) \cong \mathbb{R}^{k^{2}}$. Тогда для каждой функции $f \in C_{b}^{\mathrm{cyl}}(C([0,1], G))$ справедливо равенство

$$
\int_{C([0,1], G)} f(x) W_{G}^{z}(d x)=\lim _{\varepsilon \rightarrow 0} \frac{1}{W_{O\left(\mathbb{R}^{k}\right)}^{z}\left(C\left([0,1], G_{\varepsilon}\right)\right.} \int_{C\left([0,1], G_{\varepsilon}\right)} f_{0}(x) W_{O\left(\mathbb{R}^{k}\right)}^{z}(d x),
$$

əде $f_{0}-$ функция из $C_{b}^{\mathrm{cyl}}\left(C\left([0,1], O\left(\mathbb{R}^{k}\right)\right)\right)$, сужение которой на $C([0,1], G)$ совпадает с $f$.

Тогда для любой $f \in C_{b}^{\mathrm{cyl}}([0,1], G)$ верна цепочка равенств:

$$
\begin{aligned}
\int_{C([0,1], G)} f\left(u_{\tau}(x)\right) W_{G}^{z}(d x)=\lim _{\varepsilon \rightarrow 0} \frac{\int_{C\left([0,1], G_{\varepsilon}\right)} f\left(u_{\tau}(x)\right) W_{\mathbb{R}^{n}}^{z}(d x)}{W_{\mathbb{R}^{n}}^{z}\left(C\left([0,1], G_{\varepsilon}\right)\right)} \\
=\lim _{\varepsilon \rightarrow 0} \frac{1}{W_{\mathbb{R}^{n}}^{z}\left(C\left([0,1], G_{\varepsilon}\right)\right)}\left(\int_{C\left([0,1], \mathbb{R}^{n}\right)} \chi\left(u_{\tau}(x)\right) f\left(u_{\tau}(x)\right) W_{\mathbb{R}^{n}}^{z}(d x)\right) \\
=\lim _{\varepsilon \rightarrow 0} \frac{1}{W_{\mathbb{R}^{n}}^{z}\left(C\left([0,1], G_{\varepsilon}\right)\right)} \int_{C\left([0,1], \mathbb{R}^{n}\right)} \chi(x) f(x) \\
\quad \times e^{\tau \int_{0}^{1}(\hat{\alpha}(\tau, s) x(s), d x(s))-\frac{1}{2} \tau^{2} \int_{0}^{1}(\tilde{\alpha}(\tau, s) x(s), \tilde{\alpha}(\tau, s) x(s)) d s} W_{\mathbb{R}^{n}}^{z}(d x) \\
=\int_{C([0,1], G)} f(x) e^{\tau \int_{0}^{1}(\hat{\alpha}(\tau, s) x(s), d x(s))-\frac{1}{2} \tau^{2} \int_{0}^{1}(\tilde{\alpha}(\tau, s) x(s), \tilde{\alpha}(\tau, s) x(s)) d s} W_{G}^{z}(d x),
\end{aligned}
$$

где

$$
\tilde{\alpha}(\tau, s)=\dot{\alpha}(s) e^{-\tau \alpha(s)}, \quad \chi(x)= \begin{cases}1, & x \in C\left([0,1], G_{\varepsilon}\right), \\ 0, & x \notin C\left([0,1], G_{\varepsilon}\right) .\end{cases}
$$

Первое и четвертое равенства следуют из теоремы (и того, что $G_{\varepsilon}$ переходит в себя под действием $u_{\tau}$ ), а третье равенство следует из теоремы 1 . Доказательство закончено.

СледСтвиЕ 2. Мера Винера $W_{G}^{z}$ дифференцируема вдоль $v(x)$ и ее логарифмическая производная $\beta_{v}^{W_{G}}(x)$ равна

$$
\beta_{v}^{W_{G}}=\left.\beta_{v}^{W^{z} k^{2}}\right|_{x \in G}
$$

Доказательство аналогично следствию 1.

ЗАмечАниЕ. Аналог теоремы 2 справедлив для сферы и любого другого симметрического пространства, $\varepsilon$-окрестность которого инвариантна относительно действия $u_{\tau}$.

Автор благодарит профессора О.Г. Смолянова за постановку задачи и внимание к работе, и профессора Х. фон Вайцзеккера за полезные замечания.

\section{СПИСОК ЦИТИРОВАННОЙ ЛИТЕРАТУРЫ}

1. Smolyanov O. G., v. Weizsaecker H. // C. R. Acad. Sci. Paris. 1995. V. 321. P. 103-108. 2. Смолянов О. Г. // Докл. РАН. 1995. Т. 345. №4. С. 455-458. 3. Икэда Н., Ватанабэ С. Стохастические дифференциальые уравнения и диффузионные процессы. М.: Наука, 1986. 4. Smolyanov O. G., v. Weizsaecker H. // J. Funct. Anal. 1993. V. 118. P. 454-476. 5. CMOлянов О. Г., фон Вайцзеккер Х. // УМН. 1996. V. 51. № 2. P. 357-358. 6. Smolyanov O. G., v. Weizsaecker H. // Inf. Dim. Anal. Quant. Prob. 1999. V. 2. №1. P. 51-79. 7. Sidorova N. A., Smolyanov O. G., v. Weizsaecker H., Wittich O. The surface limit of Brownian motion in tubular neighbourhoods of an embedded Riemannian manifold. Submitted, 2003. 